\title{
A Compatibilidade Estímulo-Resposta COMO MODELO PARA O ESTUdO DO \\ COMPORTAMENTO MOTOR ${ }^{1}$
}

\author{
Luiz de Gonzaga Gawryszewski², Allan Pablo Lameira ${ }^{3}$, Fernanda \\ Maciel Ferreira ${ }^{3}$, Sabrina Guimaraes-Silva ${ }^{3}$, Erick Francisco Quintas \\ Conde $^{3}$ e Antônio Pereira Jr. ${ }^{4}$ \\ Universidade Federal Fluminense - UFF \\ Universidade Federal do Pará - UFPA
}

Quando um estímulo ocorre aleatoriamente à esquerda ou à direita do ponto de fixação, a resposta é mais rápida quando o estímulo e a tecla de resposta estão do mesmo lado (condição compatível) do que quando estão em lados opostos (condição incompatível). Na tarefa de compatibilidade espacial, observa-se uma diferença entre os Tempos de Reação Manual (TRMs) nas condições compatível e incompativel da ordem de 30-40 ms. No Efeito Simon, embora o critério para a seleção da resposta seja a forma (ou a cor) do estímulo, a posição do estímulo também influencia o TRM, facilitando-o na condição compativel e lentificando-o na incompatível. $O$ Efeito Simon pode ser invertido se a pessoa realiza testes incompatíveis previamente ao teste Simon, mostrando que associações de curto prazo, geradas pelos pareamentos incompatíveis modulam padrões de conexões estímulo-resposta baseados na memória de longo prazo.

Descritores: Controle motor. Processos motores. Tempo de reação.

Percepção espacial. Lateralidade.

1 Apoio Financeiro do CNPq, FAPERJ, CAPES, PIBIC-UFF, PROPP-UFF e UFPA.

2 Docente do Departamento de Neurobiologia - UFF. Endereço eletrônico: gawryszewski_lg@yahoo.com.br

3 Pós-Graduandos do Programa de Neuroimunologia do Departamento de Neurobiologia - UFF.

4 Docente do Departamento de Fisiologia do Centro de Ciências Biológicas - UFPA. 
L. G. Gawryszewski, A. P. Lameira, F. M. Ferreira, S. Guimaraes-Silva et al.

Neurociência cognitiva tem como objetivo estudar a relação entre fenô-
menos mentais e as estruturas neurais do cérebro humano. Para isto, técnicas sofisticadas, tais como as medidas do fluxo sangüíneo e do metabolismo cerebral através do PET (Tomografia por Emissão de Pósitrons) e da fMRI (Ressonância Magnética funcional), têm sido empregadas. Porém, as técnicas que se baseiam na medida da latência da resposta a estímulos continuam sendo instrumentos fundamentais para a compreensão dos mecanismos envolvidos com o processamento sensorial e a programação da resposta motora.

A medida do Tempo de Reação Manual (TRM) é empregada principalmente para desenvolver modelos que permitam identificar as várias etapas de processamento e para estimar o tempo envolvido em cada etapa entre a apresentação de um estímulo e a execução de uma resposta. Dependendo da complexidade do estímulo e do número de alternativas da resposta, o TRM será maior ou menor. Além disso, a relação entre as propriedades do estímulo e as propriedades da resposta acarretará respostas mais rápidas ou mais demoradas (Massaro, 1989).

Neste artigo, iremos analisar o fenômeno da Compatibilidade Estímulo-Resposta. Mais particularmente, discutiremos como as propriedades espaciais do estímulo interagem com as propriedades espaciais da resposta, facilitando ou retardando a execução da resposta. Além disso, estes resultados serão relacionados com a organização anatomo-funcional do cérebro humano, com os mecanismos de reconhecimento de partes do corpo e com interação modulatória entre as memórias visuo-motoras de breve e de longo prazo.

\section{Tempo de Transferência Inter - Hemisférica (TTIH)}

A localização do estímulo visual no espaço é uma propriedade que influencia a seleção de uma ação para este estímulo. Poder-se-ia supor que esta influência resultaria principalmente da organização anatomo-funcional do Sistema Nervoso. Na Figura 1, mostramos um esquema em que estão representados os dois hemicampos visuais, os dois hemisférios cerebrais e os dois efetores (mão esquerda e mão direita). Pode-se observar que um estímulo localizado à esquerda projeta-se para o hemisfério direito, o qual controla a mão esquerda. Da mesma forma, um estímulo localizado à direita projeta-se 
para o hemisfério esquerdo, o qual controla a mão direita. Para um estímulo localizado à esquerda desencadear uma resposta com a mão direita, é necessário que a ativação dos neurônios do hemisfério direito seja de alguma forma transferida para o hemisfério esquerdo. Isto é feito através dos sistemas de fibras comissurais que conectam os dois hemisférios cerebrais, sendo o corpo caloso a principal comissura do cérebro humano.

Considerando agora um estímulo localizado à direita, a projeção deste para o hemisfério esquerdo poderia provocar diretamente uma resposta com a mão direita (condição NÃO-CRUZADA). Por outro lado, uma resposta com a mão esquerda dependeria do cruzamento da informação do hemisfério esquerdo para o hemisfério direito, o qual, então, desencadearia a resposta com a mão esquerda (estímulo à direita - resposta com a mão esquerda - condição CRUZADA). A diferença entre os TRMs nas condições cruzada e não-cruzada corresponde ao tempo de transferência inter-hemisférica (TTIH - Poffenberger, 1912, ver revisão em Zaidel \& Iacoboni, 2003).

A medida do TTIH é feita medindo-se o TRM em 4 condições:

1. apresenta-se uma série de estímulos à esquerda para o/a voluntário/a responder com a mão esquerda (condição não-cruzada);

2. apresenta-se uma série de estímulos à direita para o/a voluntário/a responder com a mão direita (condição não-cruzada);

3. apresenta-se uma série de estímulos à esquerda para o/a voluntário/a responder com a mão direita (condição cruzada) e

4. apresenta-se uma série de estímulos à direita para o/a voluntário/a responder com a mão esquerda (condição cruzada).

As ordens destas 4 condições são alternadas entre os voluntários de modo a compensar os efeitos devidos ao cansaço e/ou treinamento. A estimativa do TTIH é feita subtraindo-se a média dos TRM nas condições cruzadas da média dos TRM nas condições não-cruzadas. Os valores comumente encontrados variam entre 1 a 5 ms (Zaidel \& Iacoboni, 2003)

Como veremos a seguir, os efeitos de Compatibilidade Estímulo-Resposta provocam, geralmente, diferenças de 20 a 40 ms, ou seja, são, aproximadamente, uma ordem de grandeza acima do TTIH (1-5 ms). 
L. G. Gawryszewski, A. P. Lameira, F. M. Ferreira, S. Guimaraes-Silva et al.

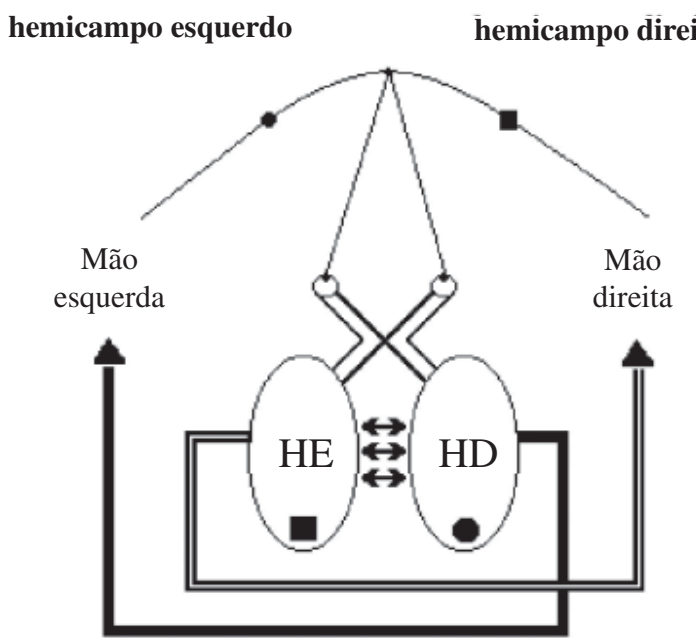

Figura 1. Esquema em que estão representados os dois hemicampos visuais, os dois hemisférios cerebrais (HE - hemisfério esquerdo; HD - hemisfério direito) e os dois efetores (mão esquerda e mão direita). Pode-se observar que um estímulo localizado à esquerda projeta-se para o hemisfério direito, o qual controla a mão esquerda. Da mesma forma, um estímulo localizado à direita projeta-se para o hemisfério esquerdo, o qual controla a mão direita.

\section{Compatibilidade Estímulo-Resposta: A Compatibilidade Espacial}

O termo Compatibilidade Estímulo-Resposta (CER ou SRC - Stimulus Response Compatibility) é usado para descrever os efeitos da correspondência entre características do estímulo e características da resposta que influenciam (facilitando ou inibindo) o TRM e a acurácia com que uma tarefa é executada. Ou seja, a presença de determinada característica do estímulo pode fazer com que os sujeitos tenham uma forte tendência em selecionar uma resposta particular em vez de outra resposta. Por exemplo, um estímulo aparecendo à esquerda induz à seleção da tecla esquerda para a resposta. Assim, a interação entre o estímulo e a resposta que produz latências menores e baixas taxas de erro é considerada mais compatível do que a interação que produz latências maiores e altas taxas de erro (Umiltà \& Nicoletti, 1990). 
Existem diferentes tipos de testes que foram empregados para estudar a questão da Compatibilidade Estímulo-Resposta. Foram estudados, entre outros os fatores: o tipo e localização dos estímulos, o tipo e localização da resposta e a instrução relevante à execução do teste. Em determinados testes, a característica relevante para a seleção da resposta é o local onde aparece o estímulo. Assim, solicita-se ao sujeito que pressione determinada tecla quando o estímulo aparecer em uma posição e outra tecla quando aparecer em outra posição. Em outros testes, a característica relevante para a seleção da resposta depende de propriedades intrínsecas ao estímulo (forma ou cor). Quando a localização do estímulo é usada para selecionar a resposta, o teste é denominado de Compatibilidade Espacial (Anzola, Bertoloni, Buchtel, \& Rizzolatti, 1977).

Como a localização de um estímulo visual no espaço influencia a seleção de uma ação para esse estímulo? A característica importante que produz esse fenômeno de compatibilidade é a correspondência espacial entre a localização do estímulo e a posição das teclas de resposta (Umiltà \& Nicoletti, 1985). As localizações são codificadas e o código da posição do estímulo é comparada com o código da posição da tecla de resposta influenciando a latência e a acurácia da resposta do sujeito. Desta forma, latências menores e baixas taxas de erros são geradas quando esses códigos são iguais e latências maiores e altas taxas de erros quando os códigos são diferentes.

\section{Compatibilidade Espacial: Posição Absoluta vs. Posição Relativa}

Os resultados obtidos por Umiltà e Nicoletti (1990) mostraram que quando a instrução dada ao/à voluntário/a era: "pressione a tecla esquerda quando um estímulo aparecer à esquerda e a tecla direita quando o estímulo aparecer à direita" (condição compatível), o TRM e o número de erros eram menores do que quando a instrução era: "pressione a tecla direita quando um estímulo aparecer à esquerda e a tecla esquerda quando o estímulo aparecer à direita" (condição incompatível).

Poder-se-ia perguntar se esta relação entre o estímulo e a tecla é estabelecida através da posição absoluta do estímulo (e da tecla) em relação à linha média do/a voluntário ou se o que importa é a posição relativa dos estímulos (e das teclas) entre si (ver revisão em Umiltà \& Nicoletti, 1990). Os resultados 
L. G. Gawryszewski, A. P. Lameira, F. M. Ferreira, S. Guimaraes-Silva et al.

mostraram que os códigos são relativos, de modo que as correspondências se dão entre os estímulos mais à esquerda (ou mais à direita) e as teclas mais à esquerda (ou mais à direita).

Estes resultados mostram que não é a divisão do campo visual em metades esquerda e direita, nem é o controle motor contralateral que determina estes fenômenos de compatibilidade estímulo-resposta.

\section{Compatibilidade Espacial: Dimensão Vertical}

Outra evidência de que a compatibilidade não é mediada pela organização anátomo-funcional dos hemisférios cerebrais humanos, per se, é a presença de compatibilidade estímulo-resposta ao longo da dimensão vertical. Nicoletti e Umiltà (1984) demonstraram que o efeito de compatibilidade espacial entre as localizações do estímulo e da resposta também existia quando os estímulos e as respostas eram verticalmente posicionados, apesar de existir um efeito mais robusto na dimensão horizontal do que na dimensão vertical.

A existência desse fenômeno de compatibilidade espacial, mesmo quando os estímulos estão dispostos verticalmente, mostra que esse fenômeno não é devido a organização anátomo-funcional dos hemisférios cerebrais, pois quando um estímulo é apresentado em cima ou embaixo do ponto de fixação central, ele ativa ambos os hemisférios, diferentemente de um estímulo disposto horizontalmente, o qual ativa um ou outro hemisfério dependendo da sua localização e a resposta pode ser executada pelo hemisfério cerebral ativado pelo estímulo ou executada pelo hemisfério oposto.

Assim, esse efeito de compatibilidade espacial ocorre porque as localizações do estímulo e da resposta geram códigos espaciais e é a formação desses códigos que determina a performance da ação, sendo esta executada mais facilmente quando esses códigos espaciais coincidem do que quando eles não coincidem. 
A Compatibilidade Estímulo-Resposta como Modelo para o Estudo...

\section{Compatibilidade Espacial: O Efeito Cruzamento}

Na Figura 2, estão representadas quatro condições relacionando a ocorrência de um estímulo à esquerda e a resposta a este estímulo (Anzola et al., 1977):

1. o estímulo ocorre à esquerda e o/a voluntário/a responde apertando a tecla esquerda empregando o dedo indicador esquerdo. Esta condição será denominada condição compatível não-cruzada. Compatível porque o estímulo e a tecla de resposta estão no mesmo lado e não-cruzada porque a mão esquerda responde apertando a tecla esquerda;

2. o estímulo ocorre à esquerda e o/a voluntário/a responde apertando a tecla direita empregando o dedo indicador direito. Esta condição será denominada condição incompatível não-cruzada. Incompatível porque o estímulo e a tecla de resposta estão em lados opostos e não-cruzada porque a mão direita responde apertando a tecla direita;

3. o estímulo ocorre à esquerda e o/a voluntário/a responde apertando a tecla esquerda empregando o dedo indicador direito. Esta condição será denominada condição compatível cruzada. Compatível porque o estímulo e a tecla de resposta estão no mesmo lado e cruzada porque a mão direita responde apertando a tecla esquerda e

4. o estímulo ocorre à esquerda e o/a voluntário/a responde apertando a tecla direita empregando o dedo indicador esquerdo. Esta condição será denominada condição incompatível cruzada. Incompatível porque o estímulo e a tecla de resposta estão em lados opostos e cruzada porque a mão esquerda responde apertando a tecla direita.

Este experimento foi importante para estabelecer uma distinção entre o efeito compatibilidade e o efeito cruzamento. Todavia, na condição cruzada, a mão esquerda pressiona a tecla à direita e a mão direita pressiona a tecla à esquerda, de forma que poder-se-ia perguntar se a lentificação da resposta é devida ao fato da mão estar localizada no hemi-espaço oposto ou ao fato da mão agir no espaço oposto. Para distinguir entre o efeito provocado pela posição da mão e o efeito devido ao local de ação da mão, Riggio, Gawryszewski e Umiltá (1986) realizaram dois experimentos nos quais cada mão era man- 
L. G. Gawryszewski, A. P. Lameira, F. M. Ferreira, S. Guimaraes-Silva et al.

tida no seu hemi-espaço, mas a ação da mão era dirigida para o hemi-espaço oposto. No primeiro experimento, os sujeitos eram testados nas 4 condições descritas acima mas em vez de cruzarem (ou não) as mãos, cruzavam (ou não) dos dedos indicadores, os quais pressionavam as teclas "V" ou "N" do teclado de um microcomputador. No segundo experimento, os sujeitos eram testados nas 4 condições descritas acima, mas em vez de cruzarem (ou não) as mãos, cruzavam (ou não) pequenos bastões que pressionavam as teclas "V" ou " $\mathrm{N}$ " do teclado de um microcomputador.

Os resultados mostraram que o cruzamento dos dedos ou dos bastões provocava o mesmo efeito que o cruzamento das mãos (Anzola et al., 1997), indicando que o efeito cruzamento ocorre quando a mão age sobre uma tecla localizada no hemi-espaço contralateral à localização anatômica da mão, e não porque a mão está localizada no hemi-espaço oposto.
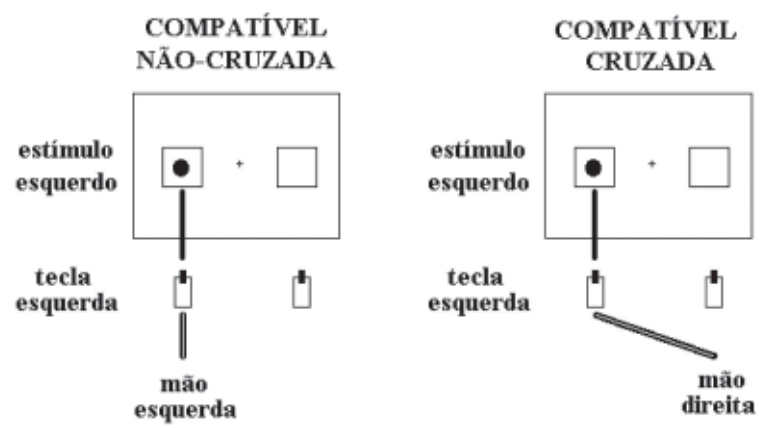

INCOMPATIVEL NÃO-CRUZADA

INCOMPATIVEL CRUZADA
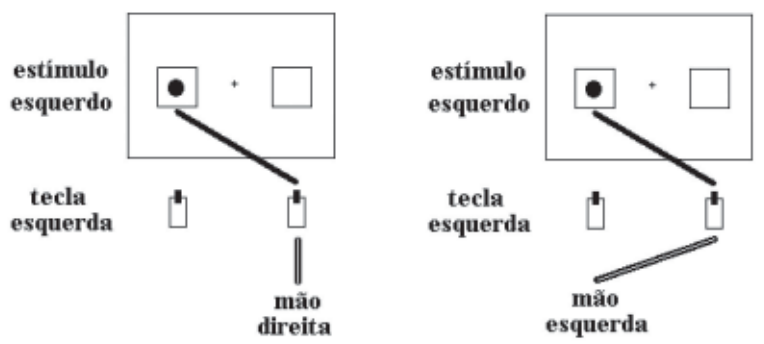

Figura 2. Esquema representando as quatro condições: Compatível não - cruzada, Compatível cruzada, Incompatível não - cruzada e Incompatível cruzada. 
É importante observar também que, o efeito de compatibilidade (estímulo e resposta do mesmo lado gerando latências menores do que estímulo e resposta em lados opostos) existia mesmo quando as mãos, dedos ou bastões estavam na posição cruzada. Isto evidencia mais uma vez que o efeito de compatibilidade não é devido a uma ativação hemisférica e sim devido à formação de códigos espaciais relacionados com as localizações do estímulo e da resposta. Assim, mesmo quando os sujeitos estavam com as mãos, dedos ou bastões na posição cruzada, a resposta ao estímulo esquerdo, por exemplo, era mais rápida quando se pressionava a tecla esquerda (condição compatível) do que quando se pressionava a tecla à direita (condição incompatível). Além disso, não existia interação entre o efeito de compatibilidade e o efeito cruzamento, indicando que eles se originam de mecanismos diferentes: relação estímulo-tecla e relação lado da mão-local de ação da mão, respectivamente (Riggio et al., 1986).

\section{Compatibilidade Estímulo-Resposta: O Efeito Simon}

Na tarefa de Simon (Figura 3), a posição do estímulo é irrelevante para a seleção da resposta, mas mesmo assim influencia no tempo de reação do sujeito. A dimensão relevante do estímulo (forma ou cor) para a seleção da resposta não tem nenhum tipo de sobreposição com nenhuma dimensão da resposta. Isto difere da dimensão irrelevante para a seleção da resposta, onde o código da localização do estímulo (hemicampo visual direito ou esquerdo) se sobrepõe ao código da localização das teclas de resposta (localização direita e esquerda). Por exemplo, figuras geométricas são apresentadas aos sujeitos e eles são instruídos a pressionar a tecla esquerda em resposta a uma figura e a tecla direita em resposta à outra figura. Os estímulos são apresentados no hemicampo visual direito ou esquerdo. Apesar da localização do estímulo ser irrelevante para a tarefa, pois o sujeito tem que reconhecer a forma para selecionar a resposta, as latências serão menores quando o lado da tecla de resposta corresponder ao lado onde o estímulo aparece do que quando estímulo e resposta estiverem em lados opostos. O mesmo é observado quando se usa estímulos com a mesma forma, mas com cores diferentes (Umiltà \& Nicoletti, 1990). 
L. G. Gawryszewski, A. P. Lameira, F. M. Ferreira, S. Guimaraes-Silva et al.

A)

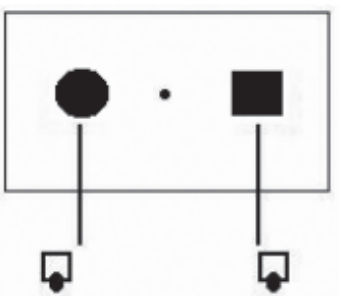

B)

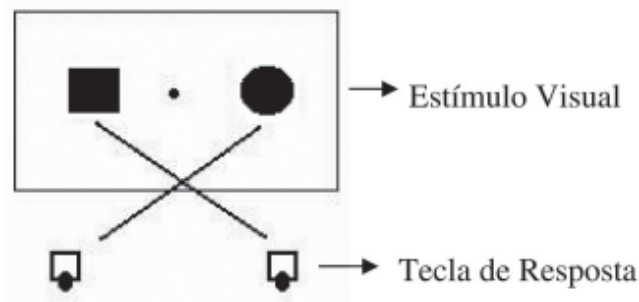

Figura 3. Tarefa de Simon. Na condição compatível (A), o estímulo está no mesmo lado da tecla de resposta e, na condição incompatível (B), o estímulo e a resposta estão em lados opostos. A dimensão relevante do estímulo (quadrado ou círculo) não tem nenhum tipo de sobreposição com nenhuma dimensão da resposta (tecla esquerda ou direita), ao contrário da dimensão irrelevante (posição esquerda ou direita).

Desta forma, a resposta correta é selecionada empregando-se uma característica não espacial do estímulo (forma ou cor), mas a localização (característica espacial) do estímulo influencia a resposta do sujeito, pois se sobrepõe à característica espacial das posições das teclas de resposta (Umiltà \& Nicoletti, 1992).

Historicamente, o Efeito Simon foi atribuído ao estágio de processamento de seleção da resposta, possivelmente devido ao fato de que efeitos da mesma natureza serem obtidos quando a localização do estímulo é a informação relevante para determinar a resposta correta (Lu \& Proctor, 1995). Em tarefas de tempo de reação de escolha onde a localização do estímulo é a informação relevante, as respostas serão mais rápidas quando o estímulo esquerdo for associado com a resposta esquerda e o estímulo direito for associado com a resposta direita do que quando essas associações forem inversas (Proctor \& Dutta, 1993). Evidências muito consistentes atribuem esse efeito de compatibilidade espacial (tarefa de compatibilidade espacial) ao estágio de seleção da resposta (Lu \& Proctor, 1995).

A maioria das hipóteses que atribuem o efeito Simon ao estágio de seleção da resposta diz que isso ocorre devido a uma competição da resposta (Umiltà \& Nicoletti, 1990). As hipóteses assumem que um código de resposta é gerado pela informação irrelevante do estímulo (sua posição) e um outro código de resposta é formado pela informação relevante (cor ou forma do estímulo). Nas situações onde os códigos de resposta formados pela informação 
relevante e irrelevante são iguais, não existe nenhuma competição e possivelmente existe até um benefício devido à redundância desses códigos de resposta. Entretanto, naquelas situações onde os códigos de resposta são diferentes, existe uma competição que deve ser resolvida antes da resposta correta ser selecionada e executada.

Essas teorias sobre o estágio de seleção da resposta assumem que um código de resposta é formado pela informação irrelevante do estímulo, porém, elas diferem nas razões propostas sobre como e porque esse código é formado (Lu \& Proctor, 1995). As três principais hipóteses que atribuem o efeito Simon ao estágio de seleção da resposta enfatizam a orientação atencional, a codificação espacial e a sobreposição dimensional entre a informação irrelevante do estímulo e a resposta, respectivamente (Umiltà \& Nicoletti, 1990).

\section{Efeito Simon: Dimensão Vertical}

Assim como o efeito de compatibilidade espacial, vários trabalhos descrevem o efeito Simon ocorrendo com um arranjo vertical dos estímulos e das teclas de resposta (Rubichi, Nicoletti, Pelosi, \& Umiltá, 2004; Rubichi, Nicoletti, \& Umiltá, 2005; Vallesi, Mapelli, Schiff, Amodio, \& Umiltá, 2005). No entanto, apesar de ocorrer uma facilitação nas resposta da condição correspondente e também uma inibição na não correspondente, algumas peculiaridades são observadas no efeito Simon vertical em comparação ao realizado na horizontal.

Vallesi et al. (2005) compararam os resultados obtidos em testes realizados na dimensão horizontal e também na vertical utilizando-se também de uma análise distribucional dos TRM e de registros encefalográficos destinados a registrar a atividade cerebral no intervalo de tempo entre a apresentação do estímulo e o início da atividade eletromiográfica (Potencial de Prontidão Lateralizado - PPL). A análise distribucional possibilitou detectar que o efeito Simon na vertical esteve presente tanto na realização dos testes mais rápidos quanto nos mais lentos. Na horizontal foi detectada uma diminuição do efeito Simon nos testes $40 \%$ mais lentos. Já, na análise do PPL, os autores verificaram um atraso para a atividade de preparação da resposta na condição não correspondente em testes verticais. 
L. G. Gawryszewski, A. P. Lameira, F. M. Ferreira, S. Guimaraes-Silva et al.

Vallesi et al. (2005) propuseram uma explicação baseada na teoria de translação para o efeito de correspondência do teste de Simon na vertical. De acordo com esse modelo teórico, tanto a posição do estímulo quanto a posição da resposta seriam cognitivamente representados por meio de códigos espaciais. Esses códigos seriam comparados durante a geração da resposta e a correspondência entre os códigos influenciaria os tempos de resposta: em situações correspondentes seriam acelerados e nas não correspondentes, lentificados.

\section{Efeito Simon: Reconhecimento de partes do corpo}

Essas tarefas de Compatibilidade Estímulo - Resposta (tarefa de Simon e de Compatibilidade espacial) servem para nos mostrar como as características do estímulo influenciam na seleção de uma ação (Lu \& Proctor, 1995). Entretanto é importante observar que selecionar uma ação para um objeto externo é um processo que envolve diferentes mecanismos neurais daqueles envolvidos com a seleção de uma ação para uma figura que representa uma parte do corpo (Tomasino, Toraldo, \& Rumiati, 2003).

A medida do TRM para determinar a lateralidade de uma figura da mão não envolve apenas o sistema visual, mas também os sistemas somestésico e motor, ou seja, é um processo no qual existe uma relação entre aquilo que o sujeito vê (figura da mão) e o que sente (a informação proprioceptiva da mão). Assim, a decisão do julgamento da lateralidade da mão é tomada através da formação da imagem mental de uma das mãos, sua projeção para a tela e a verificação da congruência ou não entre a figura na tela e a imagem da mão (Parsons, 1987; Parsons, 1994; Parsons \& Fox, 1998).

O processo de reconhecimento da lateralidade da mão está envolvido com mecanismos de auto - reconhecimento, reconhecimento e imitação de ações e aquisição de novas habilidades motoras. Levando em consideração que usamos a informação proprioceptiva para identificar a lateralidade da mão e que a localização do estímulo visual no espaço influencia na seleção de uma ação para este estímulo, uma importante questão a ser observada é como esses fenômenos de Compatibilidade Estímulo-Resposta se comportam quando códigos espaciais somestésicos-motores, relacionados com a localização e postura das mãos, são acionados pelo estímulo. 
De acordo com Lameira et al. (2004) quando a figura da mão é o estímulo relevante para a seleção da resposta existe um efeito de compatibilidade, o qual pode ser modulado por diferentes códigos espaciais/motores relacionados com a postura da mão. Nesse experimento foram apresentados desenhos de mãos no hemicampo visual direito ou esquerdo e a resposta era pressionar a tecla esquerda após o aparecimento do desenho da mão esquerda e a tecla direita após o aparecimento da mão direita. Os participantes do experimento executavam a resposta pressionando a tecla com as mãos na postura prona (palmas para baixo) e na postura supina (palmas para cima). Na postura prona, existiu o efeito Simon, porém quando os participantes estavam com as mãos na postura supina esse efeito Simon desapareceu. Esses resultados mostram uma interação entre os fenômenos de compatibilidade Estímulo - Resposta e a postura da mão.

\section{O Estudo da memória visuomotora através do tempo de reação manual}

Como já foi descrito, vemos que a diferença entre o teste de compatibilidade espacial e o teste de Simon consiste no componente relevante para realização da tarefa: posição do estímulo versus característica do estímulo. No que diz respeito às semelhanças, em ambos os testes, o efeito se baseia em uma diferença entre os TRMs na condição em que existe uma correspondência espacial entre o estímulo e a tecla de resposta e os TRMs na condição em que não existe essa correspondência.

Segundo Tagliabue, Zorzi, Umiltá e Bassignani (2000), a organização do sistema nervoso e do sistema osteo-articular, fruto da seleção natural, facilita a realização de alguns movimentos em comparação a outros. Por exemplo, existe uma tendência para utilizarmos a mão direita para alcançar um objeto localizado à direita do corpo e a mão esquerda para alcançar um objeto localizado à esquerda. Esta tendência deriva de fatores inatos que são reforçados durante o desenvolvimento. De acordo com as idéias de Tagliabue et al. (2000), nas condições de correspondência colocamos em prática algo que já estamos habituados a fazer: agir em direção aos estímulos com os efetores correspondentes. Ou seja, de forma natural costumamos a atuar nas demandas situadas à direita, no nosso ambiente externo, com algum membro (braço ou perna) 
L. G. Gawryszewski, A. P. Lameira, F. M. Ferreira, S. Guimaraes-Silva et al.

direito, pois assim é mais fácil e assim fomos acostumados a fazer desde que nascemos, pois isto garante uma eficácia maior. Portanto, consolidamos em nossa memória de procedimentos, as associações de longo prazo para as habilidades motoras de resposta com efetores correspondentes através das informações e práticas das tarefas simples do dia a dia, como, por exemplo, utilizar talheres em uma refeição. Por outro lado, nas condições não correspondentes, realizamos uma tarefa que não estamos acostumados (responder com o efetor contralateral) e para respondermos corretamente se torna necessária a constituição de associações de memórias de curto prazo não declarativas.

Tagliabue et al. (2000) decidiram estudar a interação entre as associações mnemônicas de curto e longo prazo. Para isto, estudou o efeito de um teste (compatibilidade espacial), no qual a posição do estímulo é a variável relevante para a seleção da resposta, sobre outro teste. No segundo teste (teste de Simon), a posição do estímulo é irrelevante para a seleção da resposta, a qual é determinada por outra característica do estímulo. Mais especificamente, Tagliabue et al. (2000) realizaram uma série de experimentos onde os sujeitos realizavam inicialmente o teste de compatibilidade espacial e a seguir, a tarefa de Simon. No teste de Simon, a resposta para um estímulo ocorrendo na posição correspondente é mais rápida do que para um estímulo ocorrendo na posição não correspondente. Seu objetivo principal foi averiguar se as associações de memória de curto prazo necessárias para a realização de uma tarefa de compatibilidade espacial na condição incompatível (tarefa de não correspondência) afetariam a performance nos testes correspondentes (que se baseia em informações já consolidadas) de uma tarefa tipo Simon, ou seja, o desaparecimento ou a inversão de Efeito Simon pode ser devido a testes correspondentes mais lentos e/ou a testes não-correspondentes mais rápidos. Assim, os voluntários nos experimentos de Tagliabue et al. (2000) foram divididos em 2 grupos: um grupo de sujeitos fazia um teste de compatibilidade espacial (na condição compatível) e, a seguir, uma tarefa de Simon e outro grupo realizava um teste de compatibilidade espacial (na condição incompatível) para depois serem submetidos ao teste de Simon. O primeiro experimento foi realizado com crianças e, como resultado, o grupo de crianças que praticou o teste incompatível obteve um efeito Simon reverso, ou seja, tais crianças foram mais rápidas quando não existiu correspondência entre o estímulo e a tecla de resposta, fato que não ocorreu com as crianças que tiveram como prática prévia o teste compatível. 
A Compatibilidade Estímulo-Resposta como Modelo para o Estudo...

Obedecendo ao mesmo padrão, o segundo experimento também teve como tarefa inicial o teste de compatibilidade e 24 horas depois foi realizado o teste de Simon, com a única diferença de ser realizada com adultos. Os dados do segundo experimento demonstraram que os adultos também estão sujeitos a uma alteração do efeito de correspondência através de treino prévio em uma sessão não correspondente (incompatível). Contudo, o experimento 2 não resultou em um efeito reverso como aconteceu no experimento 1. Provavelmente essa diferença se deve ao fato dos adultos terem as associações de longo prazo mais consolidadas do que nas crianças. Assim, a prática incompatível foi suficiente para eliminar o efeito de correspondência no teste de Simon mas não para reverter o fenômeno.

Resultados parecidos foram encontrados quando eles testaram o efeito Simon logo após o teste de compatibilidade espacial (sem intervalo entre as práticas) e também quando o intervalo entre os dois testes foi de sete dias. Em todos os experimentos, o grupo que teve como prática prévia as sessões compatíveis, demonstrou o efeito Simon, enquanto que o grupo que realizou testes incompatíveis não teve o efeito de correspondência, que é a base do efeito Simon.

Estes resultados mostram que o Efeito Simon pode ser invertido se a pessoa realiza testes incompatíveis previamente ao teste Simon, mostrando que associações de curto prazo, geradas pelos pareamentos incompatíveis modulam padrões de conexões estímulo-resposta baseados na memória de longo prazo.

O estudo de Tagliabue et al. (2000) utilizou apenas a dimensão horizontal para apresentação dos estímulos e também para o arranjo das teclas de respostas. Todavia, Vallesi et al. (2005) acharam diferenças significativas entre os efeitos Simon obtidos nas dimensões horizontal e vertical. Desta forma, podemos indagar se o efeito de modulação gerado pelo treino prévio em testes incompatíveis ocorreria também na dimensão vertical. Finalmente, uma questão importante refere-se à generalização do efeito. Ou seja, o que ocorreria se a tarefa de compatibilidade espacial fosse realizada em uma dimensão e o teste de Simon em uma outra, alinhada ortogonalmente?

Estudos recentes realizados no Laboratório de Neurobiologia da Atenção e do Controle Motor da Universidade Federal Fluminense (UFF) sugerem que a modulação do efeito Simon pelos pareamentos incompatíveis ocorre 
L. G. Gawryszewski, A. P. Lameira, F. M. Ferreira, S. Guimaraes-Silva et al.

seja na dimensão vertical, seja quando as tarefas são praticadas em dimensões ortogonais (Conde, Gawryszewski, \& Martins, 2006).

\section{Conclusões}

Os experimentos descritos acima mostram que a relação entre a posição do estímulo e posição da tecla de resposta (Compatibilidade EstímuloResposta) exerce uma influência importante sobre o Tempo de Reação Manual a estes estímulos. Todavia, estes vínculos espaciais entre o estímulo e a resposta não estão somente relacionados com a organização anatômica do sistema nervoso, ou seja, esta relação tem um caráter relativo e não absoluto. Além disso, estes vínculos são dinâmicos, pois podem ser modulados pelo tipo de estímulo (formas geométricas versus representações de partes do corpo) ou pelo treinamento prévio em tarefas incompatíveis, as quais influenciam a memória visuo-motora de longo prazo.

Gawryszewski, L. G., Lameira, A. P., Ferreira, F. M., Guimaraes-Silva, S., Conde, E. F. Q., \& Pereira Jr., A. (2006). Stimulus-response compatibility as a tool for studying motor behavior. Psicologia USP, 17(4), 103-121.

Abstract: When a visual stimulus is randomly displayed either to the left or to the right of the fixation point, manual response is faster when both the response key and the stimulus are located at the same side (compatible condition) than at opposite sides (incompatible condition). In a spatial compatibility task, a difference of $30-40 \mathrm{~ms}$ is found between the manual reaction times (MRT) of compatible and incompatible conditions. In the Simon effect, though the criterion to response selection is primarily stimulus shape (or color), the stimulus position also influences the MRT, either increasing it in the compatible condition or delaying it in the incompatible condition. The Simon effect can be inverted if the subject has recently performed incompatible tasks, showing that short-term memory processes elicited by the incompatible associations can modulate stimulus-response relationships based on long-term memory.

Index terms: Motor control. Motor processes. Reaction time. Spatial perception. Lateral dominance. 
Gawryszewski, L. G., Lameira, A. P., Ferreira, F. M., Guimaraes-Silva, S., Conde, E. F. Q., \& Pereira Jr., A. (2006). La compatibilité stimulusréponse comme modèle pour le étude du comportement moteur. Psicologia USP, 17(4), 103-121.

Résumé: Quand un stimulus aparait aléatoirement à gauche ou a droite du point de fixation, la réponse est plus rapide lorsque le stimulus et le levier de réponse sont du même coté (condition compatible) que lorsque ceuxci sont oposés (condition incompatible). Dans la tâche de compatibilité spatiale, la différence de temps de réaction manuel (TRMs) entre les conditions compatible et incompatible est de 1'ordre de 30-40 ms. Dans l'effet Simon, bien que le critère de selection da réponse soit la forme (ou la couleur) du stimulus, la position du stimulus influence également le TRM, produisant une facilitation dans la condition compatible et un retard dans la condition incompatible. L'effet Simon peut être inverté si le sujet réalise des testes incompatibles préalablement au test Simon. Ceci démontre que les assocations à court terme, produites par les appariements incompatibles, modulent les types de connexions stimulus-réponse basés sur la mémoire à long terme.

Mots-clés: Commande de moteur. Processes de moteur. Temps de réaction. Perception spacial. Dominance latérale.

\section{Referências}

Anzola, G. P., Bertoloni, G., Buchtel, H. A., \& Rizzolatti, G. (1977). Spatial compatibility and anatomical factors in simple and choice reaction time. Neuropsychologia, 15, 295-382.

Conde, E. Q., Gawryszewski, L. G., \& Martins, G. (2006). A compatibilidade espacial influencia o efeito simon na mesma dimensão e na dimensão ortogonal: uma análise da memória visuomotora. In Resumos de Comunicações Científicas, 21. Reunião Anual da Federação das Sociedades de Biologia Experimental - FeSBE (p. 50). Águas de Lindóia, São Paulo.

Lameira, A. P., Gawryszewski, L. G., Carvalho, A. A., Soeiro-Santos, R., Guimarães-Silva, S., Ferreira, F. M. et al. (2004). A postura da mão influencia a compatibilidade EstímuloResposta numa tarefa de reconhecimento da lateralidade da mão. In A. Ferreira, M. E. Q. Gonzalez, \& J. G. Coelho (Orgs.), Encontro com as Ciências Cognitivas (Vol. 4, pp. 153-165). São Paulo: Cultura Acadêmica.

Lu, C. H., \&. Proctor, R. W. (1995). The influence of irrelevant location information on performance: A review of the Simon and Spatial Stroop effects. Psychonomic Bulletin \& Review, 2, 174-207. 
L. G. Gawryszewski, A. P. Lameira, F. M. Ferreira, S. Guimaraes-Silva et al.

Massaro, D. W. (1989). Experimental Psychology: An information processing approach. Orlando, FL: Harcourt Brace College.

Nicoletti, R., \& Umiltá, C. (1984). Right - left prevalence in spatial compatibility. Perception and Psychophysics, 35, 333-343.

Parsons, L. M. (1987). Imagined transformation of one's hands and feet. Cognitive Psychology, 19, 178-241.

Parsons, L. M. (1994). Temporal and kinematic properties of motor behavior reflected in mentally simulated action. Journal of Experimental Psychology: Human Perception and Performance, 20, 709-730.

Parsons, L. M., \& Fox, P. T. (1998). The neural basis of implicit movements used in recognising hand shape. Cognitive Neuropsychology, 15, 583-615.

Poffenberger, A. T. (1912). Reaction time to retinal stimulation, with special reference to the time lost in conduction through nerve centers. Archives of Psychology, 23, 1-73.

Proctor, R. W., \& Dutta, A. (1993). Do the same stimulus-response relations influence choice reactions initially and after practice? Journal of Experimental Psychology: Learning, Memory, and Cognition, 19, 922-930.

Riggio, L., Gawryszewski, L., \& Umiltá, C. (1986). What is crossed in crossed hands effects? Acta Psychologica, 62, 89-100.

Rubichi, S., Nicoletti, R., \& Umiltá, C. (2005). Right-left prevalence effect with task irrelevant spatial codes. Psychological Research, 69, 167-178.

Rubichi, S., Nicoletti, R., Pelosi, A., \& Umiltá, C. (2004). Right-left prevalence effect with horizontal and vertical effectors. Perception and Psychophysics, 66, 255-263.

Tagliabue, M., Zorzi, M., Umiltá, C., \& Bassignani, F. (2000). The role of long-term-memory and short-term-memory in the Simon effect. Journal of Experimental Psychology: HumanPerception and Performance, 26, 648-670.

Tomasino, B., Toraldo, A., \& Rumiati, R. I. (2003). Dissociation between the mental rotation of visual images and motor images in unilateral brain-damaged patients. Brain \& Cognition, 51, 368-371.

Umiltá, C., \& Nicoletti, R. (1985). Attention and coding effects in S-R compatibility due to irrelevant spatial cues. In M. I. Posner \& O. S. M. Marin (Eds.), Attention and performance XI (pp. 457-471). Hillsdale, NJ: Erlbaum.

Umiltá, C., \& Nicoletti, R. (1990). Spatial stimulus-response compatibility. In R. W. Proctor \& T. G. Reeve (Eds.), Stimulus-response compatibility: An integrated perspective (pp. 89-116). Amsterdam: Elsevier. 
Umiltà, C., \& Nicoletti, R. (1992). An integrated model of the Simon effect. In J. Alegria, D. Holender, J. Junca de Moraes, \& M. Radeau (Eds.), Analytic approaches to human cognition (pp. 331-350). Amsterdam: Elsevier.

Vallesi, A., Mapelli, D., Schiff, S., Amodio, P., \& Umiltá, C. (2005). Horizontal and vertical Simon effect: Different underlying mechanisms? Cognition, 96, B33-B43. Erratum in: Cognition (2005), 96, B115.

Zaidel, E., \& Iacoboni, M. (2003). Introduction: Poffenberger's simple reaction time paradigm for measuring interhemispheric transfer time. In E. Zaidel \& Iacoboni (Eds.), The parallel brain (pp. 1-7). Cambridge, MA: The MIT Press.

Recebido em: 28.11.2006

Aceito em: 20.12.2006 